\title{
Research Progress on Environmental Impact Assessment of Urban Construction Activities
}

\author{
CUI Songxu ${ }^{1}$, GONG Zhiqi ${ }^{1,2^{*}}$ \\ ${ }^{1}$ School of Civil Engineering, Qinghai University,810016, Xining, Qinghai, China. \\ ${ }^{2}$ Qinghai Provincial Key Laboratory of Energy-saving Building Materials and Engineering Safety, 810016, Xining, Qinghai, China.
}

\begin{abstract}
The process of building new construction, reconstruction, and demolition has a very significant environmental impact. As the pillar industry of the national economy, the construction industry has put tremendous pressure on the environment in its production process. This pressure is not only manifested in the consumption of resources and energy, but also in the discharge of pollutants in the three states of solid, liquid and gas. This article aims to sort out the environmental impact assessment methods of construction activities at home and abroad, and sort out the research progress of environmental impact assessment. First, start from the macro background to explain the necessity of systematic analysis and evaluation of the environmental impact in the construction field, and then briefly comb the environmental impact assessment methods in the construction field at home and abroad from the aspects of applied research, advantages and limitations, and finally summarize and look forward to the article. It can provide scholars with a little reference and reference, and promote the further expansion and improvement of environmental impact assessment methods in the construction field.
\end{abstract}

\section{Introduction}

As an important industry of the national economy, the construction industry has a very important impact on the sustainable development of the environment, society and economy. Every year, large-scale urban construction activities bring convenience to people, but also produce relatively serious environmental impacts and a large amount of resource and energy consumption, which to a certain extent hinders the realization of sustainable development. If a building is regarded as a product, then the whole life cycle of the building is from raw material mining-transportation-processing-transportation-

construction-operation and maintenance-demolition and disposal. The ecological environment brings greater pressure. This pressure is not only reflected in the supply of resources and energy, but also in the absorption of pollutants produced in each of the above-mentioned links. These pollutants may be discharged during energy production. The various types of greenhouse gases, toxic and harmful gases, sewage and solid waste, may also be solid, liquid, and gas three types of pollutants produced during the production of construction materials, building construction, renovation, and dismantling. According to the "China Building Energy Consumption Research Report" issued by the China Building Energy Conservation Association in 2017, China's building energy consumption was 947 million tons of standard coal, accounting for $21.10 \%$ of the country's total energy consumption, and building carbon emissions were 1.96 billion tons, accounting for $19.5 \%$ of the national energy carbon emissions [1]. The European Commission mentioned in the Building Energy Performance Directive in 2010 that the construction industry accounts for about $40 \%$ of the EU's total energy consumption [2]. In addition, studies have shown that as of 2015, the total amount of construction waste in my country has reached 7 billion tons, and the new construction waste generated annually exceeds 400 million tons [3]. From these data, the construction industry is a major challenge for us to reduce environmental pollution, achieve sustainable development, and move toward a low-carbon economy. Therefore, a systematic assessment of the environmental impact of urban construction activities is very necessary.

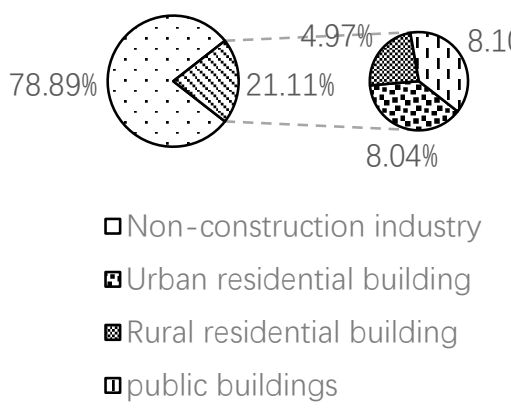

Fig1. The proportion of China's building energy consumption in 2017(The data are from China building Energy Consumption research report)

\footnotetext{
"Corresponding author E-mail address: 442953016@qq.com
} 


\section{Status of research on environmental impact assessment of construction activities}

In response to the inevitable environmental problems caused by the construction industry, scholars have established corresponding associations, scientific research groups, and released relevant research methods and evaluation tools to alleviate or even eliminate this environmental problem. According to the literature review, the environmental evaluation system can be divided into: clause-based green building evaluation system, material flow analysis method, LCA and integrated evaluation method based on LCA idea. Next, we will briefly sort out the environmental impact assessment methods in the construction field at home and abroad from the aspects of applied research, advantages and limitations.

\subsection{Ecological Footprint}

The ecological footprint (EF) refers to the biologically productive area that is necessary to maintain a person, region, or country for survival or that can accommodate human wastes [4,5]. By comparing the ecological footprint demand with the carrying capacity of the natural ecosystem (also known as the ecological footprint supply), the sustainable development status of a country or region can be quantitatively judged, so as to make scientific plans and plans for future human survival and social and economic development. Suggest.

The calculation of the ecological footprint is based on two simple facts: 1 . We can retain most of the resources consumed and most of the waste generated; 2. Most of these resources and waste can be converted into biological production that can provide these functions Sex land. First, the type of biological production area and its balancing treatment are carried out. The purpose of this stage is to convert these biological production areas with different ecological productivity into areas with the same ecological productivity, so as to summarize the ecological footprint and ecological carrying capacity; then proceed to the ecological per capita Footprint component calculation. This stage is to convert different energy consumption into a certain type of energy land area, that is, to estimate the land area required by natural assets based on the consumption rate of one of the energy sources; then summarize the ecological footprint; secondly, calculate the ecological Carrying capacity; the last is to judge whether it is an ecological deficit or an ecological surplus by the magnitude of the two values.

Domestic scholars have conducted various applied researches based on the ecological footprint method. $\mathrm{Xu}$ Zhongmin et al. [6] conducted a dynamic analysis of the ecological footprint, calculated the ecological footprint of Gansu Province in 1998, and quantitatively judged whether it was within the range of ecological carrying capacity; Liu Miao [7] based on the ecological footprint, analysed the ecological footprint of the upper reaches of the Minjiang River. Carrying capacity and landscape pattern changes were evaluated and predicted; Li Dan et al. [8] evaluated the ecological carrying capacity of the Yalong River source area, replaced the fossil energy land with woodland and grassland, and replaced it with a certain proportion. The final calculation More similar to the source area. Zhang Sheng et al. [9] established a multidrive evaluation model to evaluate the water ecological carrying capacity of the Beijing-Tianjin-Hebei region; Gao Wei et al. [10] optimized the water ecological carrying capacity model of the basin based on the coupling process of water quantity and water quality. And studied the water ecological carrying capacity of the Panlong River Basin.

The ecological footprint method analysis index provides a "fair stalk" for measuring the degree of sustainability. It can objectively measure and compare the degree of sustainability in two dimensions in time and space, so that people can clearly know the sustainability of reality. How far is the goal, so as to help monitor the effectiveness of the implementation of sustainable programs. However, the calculation results can only reflect the impact of economic decisions on the environment, without paying attention to the energy consumption of ecological products and ecological services. At the same time, it also ignores other important factors in the development and utilization of resources, such as the promotion of industrial urbanization and the occupation of cultivated land due to pollution Land degradation caused by, erosion, etc. [11,12].

\subsection{Material flow analysis}

Material flow analysis refers to the systematic analysis of material flow and storage in a specific system within a certain time and space. It mainly involves the source, path and sink of material flow. According to the law of conservation of mass, the results of material flow analysis can always achieve the final material balance through all its input, storage and output processes [13].

The basic idea of the material flow analysis method can be traced back to many different research fields more than 100 years ago [13]. In the field of economics, Leontief proposed the input-output balance sheet in the 1930s [14,15]. Research on resource protection and environmental management first appeared in the 1970s, and these two initial application areas were: (1) urban metabolism; (2) analysis of pollutant migration paths in watersheds or urban areas [13]. Later, the theory of material balance, industrial metabolism, etc. laid a theoretical foundation for the application of material flow analysis. Austria, Japan, and Germany were the first to use material flow analysis to analyse the natural resources and material flow in their own economic systems [16], which marked the beginning of large-scale application research of material flow analysis worldwide. Since 1997, the World Resources Institute has conducted a comprehensive analysis of the material flow in the economic systems of five countries (the United States, Japan, Austria, Germany, and the Netherlands). At the same time, more and more countries have begun to use material flow analysis to conduct a comprehensive analysis and evaluation of their own economic systems, such as China, the United 
Kingdom, Sweden, and Italy. In 2004, Paul H. and others co-authored the book Practical Handbook of Material Flow Analysis, which systematically introduced the concept, process, application scope and goals of material flow analysis, and stated the specific operation methods of its database management and software application. And listed many classic cases on environmental management, resource protection, waste management and regional material flow analysis and management.

Because the environment in which the economic system is located is a complex nonlinear system, and its behaviour is difficult to predict, it is difficult to establish a model to analyse it to find the results and mechanism of its change. The material flow analysis method can effectively analyse the material flow of the system from the perspective of the input-output balance sheet. Through analysis, we can get concise environmental pressure and sustainable development indicators. Of course, the material flow analysis method also has some shortcomings. The current material flow analysis only considers the quality of the material and ignores the different environmental impacts that different material flows may bring, which weakens the link between the material flow index and the environmental impact caused by the material flow. The material flow analysis method cannot determine what should be reduced in order to achieve sustainable development? This key issue. Furthermore, the purpose of the material flow analysis method is to quantitatively study the physical changes of the total material mass passing through the interface of the economy and the environment, and does not include the material flow in the economic system such as the transfer of products in different sectors, and the economic The system is treated as a "black box" [16, 17]. In addition, the data needed for material flow analysis in the economic system can almost be found at the national scale, but the relevant data at the small regional scale is often due to some objective reasons (such as the limitation of statistical content, the monopoly of large local companies and enterprises, etc.) It is difficult to obtain, and this also limits the application of material flow analysis methods in economic systems.

\subsection{Green Building Evaluation System}

Green building evaluation refers to the evaluation of the green environmental protection of the building through a series of standardized measures and evaluation processes to achieve the goal of green building. Since the 1990s, many countries in the world have established their own green building evaluation systems. However, due to differences in the level of economic development and construction industry development in different countries, as well as differences in geographic location, topography, history and culture, different countries When formulating a green building evaluation system, specific details such as the importance of evaluation indicators, the level of detail of evaluation provisions, and the stage integrity of the evaluation system are not the same [18]. The current green building evaluation system is as follows:

(1) Building Research Establishment Environmental
Assessment Method. This method was proposed in 1990 by the British Institute of Architecture. The main evaluation objects are the new construction of residential buildings, ecological buildings, public buildings, industrial buildings, and the reconstruction of existing buildings. The evaluation content covers management, energy, water, materials, ecology, land and ecology, transportation, pollution, building comfort, innovation, etc There are four evaluation levels: Excellent, Excellent, Good, and Passed.

(2) Green Building Challenge. In 1998, scholars from more than ten countries including Canada researched and proposed. The main assessment objects are residential buildings, office buildings, and school buildings that are newly built and rebuilt from existing buildings. The evaluation content involves resource consumption, indoor air quality, environmental load, economy, environmental sustainability, maintainability, and operation management. A five-level scoring system has been established.

(3) Leadership in Energy and Environmental Design. The US Green Building Council proposed the LEED system in 1998. The objects to be assessed are residential buildings and public buildings that are newly built and rebuilt from existing buildings. The evaluation content involves sustainable sites, water conservation, energy and atmosphere, materials and resources, indoor environmental quality, location and transportation, innovative design, and regional priority. The total number of projects is $69,26-32$ points are certified, $33-38$ points are silver, 39-51 points are gold, and 52-69 points are platinum.

(4) Comprehensive Assessment System for Building Environmental Efficiency. In the context of the general trend of the sustainable development concept, in April 2001, the "Comprehensive Building Environmental Assessment Research Committee" was jointly established by industry (enterprises), politics (government), and academics (scholars) in Japan, and jointly launched the project research, and finally developed "building comprehensive environmental performance evaluation system"-CASBEE. The assessment objects are buildings of various purposes and scales that are newly built and rebuilt from existing buildings. The assessment content involves the performance and quality of the building environment (including indoor environment, service facilities and outdoor environment) and the load of the building environment (including resources, energy, materials, and off-site environment). A total of 1 to 5-star ratings.

In addition, there are the Canadian system, the Chinese system, the Australian and German systems and so on.

\subsection{LCA}

Life Cycle Assessment, referred to as LCA, is aimed at the entire life cycle of a product (building) - that is, the entire process from the extraction and transportation of raw materials to the design, manufacture, use, recycling and final disposal of the product, quantitative calculation and analysis of the product Actual and potential energy and resources consumed and environmental management tools 
corresponding to pollutants discharged [19]. The earliest use of LCA ideas for environmental impact assessment began in the United States and other Western countries in the 1970s. For example, the well-known Coca-Cola Company conducted quantitative analysis of the environmental resource utilization and environmental emissions of its packaging products. After the efforts of many scholars, organizations, and scientific research groups, some basic frameworks, databases, and the more famous evaluation software SimPro appeared one after another. At this time, people realized that a unified international name and standard was needed for better research and communication. The name LCA has been fixed internationally. In 1997, with the joint efforts of international scholars, the International Organization for Standardization issued the first standard of life cycle theory-ISO14040 "Life Cycle Assessment-Principles and Framework", and then successively issued ISO 14041 "Life Cycle Assessment- Purpose and scope determination, inventory analysis, ISO14042 "Life Cycle AssessmentImpact Assessment", ISO14043 "Life Cycle AssessmentInterpretation of Results", etc. International standards are of great significance to the research and application of LCA theory. Before and after the standards were published one after another, scholars have achieved positive results for LCA research.

LCA is composed of 4 interrelated parts, namely: purpose definition and scope determination, inventory analysis, impact evaluation, and result interpretation. Purpose definition and scope determination are the first steps in life cycle assessment. It is the starting point and foothold of all subsequent steps. At this stage, it is necessary to clearly put forward the purpose and background of the analysis, as well as point out the assumptions and constraints involved in the analysis, and set functional units [19].

The inventory analysis stage is usually the most timeand labour-intensive stage in life cycle assessment [20,21], because of the lack of data related to the research object. In general, it is easier to collect data than reference search data [22]. Therefore, many databases have been developed in recent years. Foreign countries are particularly prominent in this regard. Related software and databases have been developed towards commercialization, such as SimPro, GaBi, etc. Research in my country started relatively late, in which $\mathrm{Xu}$ Xiaoming [23] introduced a logical hierarchical connection environmental engineering database design method, that is, based on hierarchical analysis, based on connection relations, combined with object-oriented technology methods for environmental engineering database design; Liu Xialu [24] summarized and put forward the basic method of establishing the life cycle reference database in my country; Gong Xianzheng, Li Xiaoqing and others [25-27] developed the China Material Environment Database (SinoCenter) using ASP.NET and SQL Server, and established the China Material Life Cycle Analysis Database platform. Life cycle analysis is a widely used environmental management tool. Its specific application requires the support of relevant environmental load data and evaluation method system data at different levels, regions and different technical levels. The reliability of data directly affects the results of product life cycle assessment Credibility and application performance. Therefore, all countries in the world are actively constructing LCA databases in line with their own actual conditions, and have achieved good results. In recent years, the construction of LCA databases in my country has slowly caught up.

Impact evaluation is the more difficult and technical part of LCA, and its methodology and evaluation model are one of the focuses of scholars in this field [19]. The general steps of impact evaluation are: classification, characterization, standardization, and weighting. The purpose of life cycle impact assessment (LCIA) is to understand and evaluate potential environmental impacts, including their scale and importance, and result interpretation is to summarize the results of inventory analysis and impact assessment to form corresponding conclusions and recommendations. In the life cycle assessment, if the scope of the investigation, the definition and distribution of the system boundary in the inventory analysis are different, the data sources are different, the impact evaluation is characterized, and the weighting method is different, different conclusions will be produced. Therefore, it is necessary to explain the necessary results after the evaluation.

\subsection{Integrated evaluation method based on LCA idea}

Although LCA is currently a widely used environmental management tool, it still has some limitations. For LCA to obtain reliable and application-performing evaluation results, it needs massive and high-quality data support; the endpoints of the LCA evaluation system are a regional (or local) indicator, which requires corresponding geographic information (population). Density, economic level, geology, landform, etc.) cooperate with the interpretation results [28], so that the interpretation of the impact evaluation results has practical significance. Therefore, scholars have begun to try to combine LCA with information technology, and make up for the shortcomings of the LCA method by incorporating the element of GIS. Domestic scholar Liu Fang et al. [29,30] believe that to solve the problem of the lack of traditional LCA spatial location data, geographic information systems should be introduced to achieve the acquisition of spatial location data. He successively published articles discussing the necessity and possibility of introducing spatial location data into LCA, and proposed a new functional structure and database framework of product material management information system. Tian Yajun et al. [28] reviewed the progress of the regionalization of life cycle assessment, discussed the necessity and feasibility of introducing spatial information data into LCA, and the important role of GIS in the regionalization of LCA. Wang Xue [31] believes that the coupling of GIS and LCA can well supplement, improve and manage traditional data, help explore the spatial characteristics of the environmental impact of products, activities or processes, and conduct environmental impact assessments related to land use. 
Some foreign scholars also believe that GIS should be integrated. Zea Escamilla E [32] researched that the use of geographic information system makes the development of building materials and LCIA characteristic data of buildings highly consistent. Geyer et al. [33] believe that geographically related factors play a very important role in specific cases of building life cycle assessment, but they are difficult to solve in life cycle assessment. When using geographic information system technology, factors such as land use, seismic hazard areas, and material transportation distances are very straightforward. Gao Yu [34] studied the use of BIM model construction, the application of LCA method to calculate the environmental impact of building materials or components, and the spatial analysis of the obtained data through buffer analysis in GIS and network analysis technology; Lu Lu et al. [35] proposed a technical method of coupling geographic information system (GIS) spatial analysis and life cycle analysis (LCA) to assess the development and utilization potential of energy plants, and found that the spatial grid evaluation method can provide more refined data support for scientific decision-making. Foreign scholars Hiloidhari et al. [36] combined GIS and LCA research methods to demonstrate the role of spatial LCA in agricultural waste residue bioenergy planning. Saad et al. [37] established regionalized environmental impact characteristic factors for soil ecosystems in different ecological regions of Canada. Mutel et al. [38] calculated the difference in ecotoxicity effects produced by power plants in different locations in the United States based on GIS tools. Österbring M et al. [39] constructed a geographic information system model based on a specific building for a multi-family residential building in Gothenburg, and used an exploratory LCA method to evaluate the environmental impact of the renovation. S. GARCÍA-PÉREZ et al. [40] used geographic information systems combined with life cycle assessment methods to characterize and evaluate urban areas from the perspective of energy efficiency.

GIS is a technical system composed of three parts: data input, storage, editing, spatial analysis and spatial query, visual expression and output. Using GIS technology to analyse the life cycle of a product can describe complex spatial and attribute data in the form of a map, and realize spatial visualization of environmental impact. In addition, it can further demonstrate the temporal and spatial distribution of environmental impacts of urban construction activities in different research periods, which can provide management methods for environmental quality management in the construction industry and realize refined management of the built environment.

\section{Conclusion}

There are many environmental impact assessment tools and methods. The clause-based green building evaluation system has obvious advantages in evaluating some indicators that are difficult to quantify; the material flow analysis method is aimed at the total material quality at the interface between the economy and the environment in a larger area Quantitative research is carried out on the physical changes of the country, but it cannot determine what should be reduced in order to achieve sustainable development? Moreover, the current analysis is mostly based on the national scale, and it is impossible to obtain sufficient statistical data at the urban scale due to objective reasons; the ecological footprint method only calculates the supply balance of land resources and petrochemical energy materials, and does not involve other environmental impacts. Therefore, it is not dominant in many environmental impact studies; the evaluation system based on the LCA idea can not only perform effective quantitative analysis and evaluation of the current environmental impact, but also provide a comprehensive evaluation of the building and its "cradle to grave". The environmental issues involved in the process are evaluated, but the data requirements are particularly high and the necessary geographic information is lacking. Therefore, further research on the integrated application of the two methods of coupling LCA and GIS is needed.

\section{Acknowledgements}

This study was supported by the Qinghai Provincial Applied Basic Research Project Fund (Grant No.2018-ZJ734) , the National Natural Science Foundation of China (Grant No.71463047) and the Qinghai Provincial Key Laboratory of Energy-saving Building Materials and Engineering Safety (Grant No. 2018-ZJ-T01).

\section{References}

1. China Building Energy Association, Architecture.J.7 30 (2020)

2. Parliament, European. Official journal of the European union 13 (2010)

3. Xiao Xuwen,Feng Dakuo,Tian Wei,Const.Tech.J. 44 6-8 (2015)

4. Ress W E. Focus 6 121-130 (1996)

5. China Commission for International Cooperation on Environment and Development, World Environment 52-57 (2008)

6. Xu Zhongmin, Zhang Zhiqiang, Cheng Guodong. Acta Geographica Sinica 55 607-616 (2000)

7. Liu Miao. Shenyang Institute of Applied Ecology, Chinese Academy of Sciences,(2007)

8. Li Dan, Huang Chuanyou, Yin Tong, Hydropower Energy Science 36 38-41 (2018)

9. Zhang Sheng, Wang Tieyu, Zhang Hong, Acta Ecologica Sinica 37 4159-4168 (2017)

10. Gao Wei, Yan Chang'an, Li Jincheng, Acta Scientiae Circumstantiae,37 755-762 (2017)

11. Qu Xiuqi, Liu Miao, Li Chunlin, Hu Yuanman, Journal of Meteorology and Environment,35 113-119 (2019)

12. Li Tao, Gong Yabo Journal of Nanhua University (Natural Science Edition) 34 60-67+96 (2020)

13. Paul H, Helmut R Practical Handbook of Material Flow Analysis (2004) 
14. Leontief W. International Arts and Sciences Press(1977)

15. Leontief W. Input-Output Economics Oxford University Press, (1966)

16. Huang Heping, Bi Jun, Zhang Bing, Acta Ecologica Sinica, 27 368-379 (2007)

17. Zhang Ling, Yuan Zengwei, Bi Jun. Acta Ecologica Sinica 29 6189-6198 (2009)

18. Zhao Hongjia. Jilin University of Architecture, 2019.

19. Zheng Xiujun, Hu Bin. Progress in Science and Technology 30 155-160 (2013)

20. Rebitzer, G., Ekvall, T., Frischknecht, R. Environmental International 30 701-720 (2004)

21. Finnveden, G., Hauschild, M. Z., Ekvall, Journal of Environmental Management 91 1-21 (2009)

22. Baumann, H. , Tillman, A-M Lund University (2004)

23. $\mathrm{Xu}$ Xiaoming, Mao Zhiwei, Rong Kangquan, Mechanical and Electrical Engineering Technology 36 15-17 (2010)

24. Liu Xialu, Wang Hongtao, Chen Jian, Journal of Environmental Sciences 30 2136-2144 (2010)

25. Gong Xianzheng, Zhang Qun, Liu Yu, Journal of Beijing University of Technology 35 997-1001 (2009)

26. Gong Xianzheng, Nie Zuoren, Wang Zhihong, Progress in China Materials 30 1-7 (2011)

27. Li Xiaoqing, Gong Xianzheng, Nie Zuoren, Progress in Chinese Materials 35 171-178 (2016)

28. Tian Yajun, Deng Yelin, Zhang Yueling, Xie Kechang, CIESC Journal 37 2195-2201 (2016)

29. Liu Fang, Shi Jinfa, Lu Changde, Journal of Xinyang Normal University 23 467-469 (2010)

30. Liu Fang, Shi Jinfa, Lu Changde, Journal of Nanjing University of Aeronautics and Astronautics 43 91-94 (2011)

31. Wang Xue, Shi Xiaoqing, Acta Ecologica Sinica 37 1346-1357 (2017)

32. Edwin Zea Escamilla, Guillaume Habert, The International Journal of Life Cycle Assessment 22 1210-1219 (2017)

33. Geyer R, Stoms DM, Lindner JP, International Journal of Life Cycle Assessment 15 454-467 (2010)

34. Gao Yu, Shenzhen University, 2018.

35. Lu Lu, Fu Xinyu, Jiang Dong, Journal of GeoInformation Science 16 328-334 (2014)

36. Hiloidhari, M.; Baruah, D. C.; Singh, A.; Kataki, S.; Medhi, K.; Kumari, S.; Ramachandra, T. V.; Jenkins, B. M.; Thakur, I. S., Bioresource Technology 242 (2017)

37. Saad R, Margni M, Koellner T, Int. J. LCA 16 198211 (2011)

38. Mutel L, Pfister S, Hellweg S. Environ. Sci. Technol.,46 1096-1103 (2011)

39. Magnus Österbring, Érika Mata, Liane Thuvander, Building and Environment 165 1-8 (2009)
40. S. García-Pérez, J. Sierra-Pérez, J. BoschmonartRives, International Journal of Sustainable Development and Planning 2 294-303 (2017) 